Acta Crystallographica Section E

Structure Reports

Online

ISSN 1600-5368

\section{N-(2-Aminoethyl)-5-(dimethylamino)- naphthalene-1-sulfonamide}

\section{Shi-lei Zhang, ${ }^{\text {a* }}$ Bi-lin Zhao, ${ }^{a}$ Zhen-hong Su, ${ }^{b}$ Xian-you $\mathrm{Xia}^{\mathrm{a}}$ and Yong Zhang ${ }^{\mathrm{a}}$}

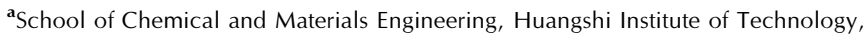
Huangshi 435003, People's Republic of China, and 'bedical School, Huangshi Institute of Technology, Huangshi 435003, People's Republic of China

Correspondence e-mail: zy0340907@yahoo.com.cn

Received 4 May 2009; accepted 23 May 2009

Key indicators: single-crystal X-ray study; $T=298 \mathrm{~K}$; mean $\sigma(\mathrm{C}-\mathrm{C})=0.003 \AA$ $R$ factor $=0.041 ; w R$ factor $=0.107 ;$ data-to-parameter ratio $=16.4$.

In the title compound, $\mathrm{C}_{14} \mathrm{H}_{19} \mathrm{~N}_{3} \mathrm{O}_{2} \mathrm{~S}$, the $\mathrm{N}$ atom of the dimethylamino group and the $\mathrm{S}$ atom are displaced by 0.078 (2) and 0.084 (2) $\AA$, respectively, from the naphthalene ring plane. The 2-aminoethyl group has a coiled conformation with an $\mathrm{N}-\mathrm{C}-\mathrm{C}-\mathrm{NH}_{2}$ torsion angle of $53.6(4)^{\circ}$. In the crystal structure, intermolecular $\mathrm{N}-\mathrm{H} \cdots \mathrm{N}$ and weak $\mathrm{C}-$ $\mathrm{H}$... O hydrogen bonds link molecules into chains along [001].

\section{Related literature}

For applications of ligands containing the 5-(dimethylamino)naphthalene-1-sulfonyl (dansyl) group, see: Corradini et al. (1996, 1997); Christoforou et al. (2006).

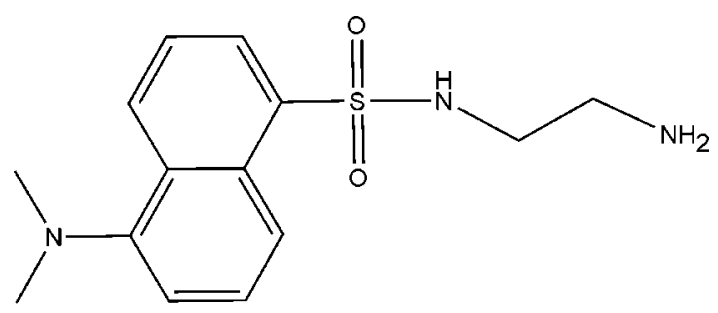

\section{Experimental}

Crystal data

$\mathrm{C}_{14} \mathrm{H}_{19} \mathrm{~N}_{3} \mathrm{O}_{2} \mathrm{~S}$

$M_{r}=293.38$

Orthorhombic, Pna2 $a=15.5221(15) \AA$

$$
\begin{aligned}
& b=11.5423(11) \AA \\
& c=8.1360(8) \AA \\
& V=1457.7(2) \AA^{3} \\
& Z=4
\end{aligned}
$$

Data collection

Bruker SMART CCD diffractometer

Absorption correction: multi-scan (SADABS; Sheldrick, 1997)

$T_{\min }=0.956, T_{\max }=0.956$

Refinement

$R\left[F^{2}>2 \sigma\left(F^{2}\right)\right]=0.041$

$w R\left(F^{2}\right)=0.107$

$S=1.11$

3140 reflections

192 parameters

1 restraint $\mu=0.23 \mathrm{~mm}^{-1}$

$T=298 \mathrm{~K}$

$0.20 \times 0.20 \times 0.20 \mathrm{~mm}$

7478 measured reflections 3140 independent reflections 3012 reflections with $I>2 \sigma(I)$ $R_{\text {int }}=0.029$

$\mathrm{H}$ atoms treated by a mixture of independent and constrained refinement

$\Delta \rho_{\max }=0.24{\mathrm{e} \AA^{-3}}^{-3}$

$\Delta \rho_{\min }=-0.25{\mathrm{e} \AA^{-3}}^{-3}$

Absolute structure: Flack (1983), 1332 Friedel pairs

Flack parameter: -0.03 (8)
Mo $K \alpha$ radiation

Table 1

Hydrogen-bond geometry $\left(\AA{ }^{\circ}\right)$.

\begin{tabular}{lllll}
\hline$D-\mathrm{H} \cdots A$ & $D-\mathrm{H}$ & $\mathrm{H} \cdots A$ & $D \cdots A$ & $D-\mathrm{H} \cdots A$ \\
\hline $\mathrm{C} 6-\mathrm{H} 6 \cdots \mathrm{O} 1$ & 0.93 & 2.48 & $3.093(3)$ & 123 \\
$\mathrm{~N} 3-\mathrm{H} 3 A \cdots \mathrm{N} 2$ & $0.88(5)$ & $2.52(6)$ & $2.972(4)$ & $113(4)$ \\
$\mathrm{C} 11-\mathrm{H} 11 \cdots \mathrm{O} 1{ }^{\mathrm{i}}$ & 0.93 & 2.49 & $3.146(3)$ & 128 \\
$\mathrm{~N} 2-\mathrm{H} 2 D \cdots \mathrm{N} 3{ }^{\text {ii }}$ & $0.87(3)$ & $2.02(4)$ & $2.869(4)$ & $163(3)$
\end{tabular}

Symmetry codes: (i) $x, y, z+1$; (ii) $-x+2,-y+1, z-\frac{1}{2}$.

Data collection: SMART (Bruker, 2007); cell refinement: SAINTPlus (Bruker, 2007); data reduction: SAINT-Plus; program(s) used to solve structure: SHELXS97 (Sheldrick, 2008); program(s) used to refine structure: SHELXL97 (Sheldrick, 2008); molecular graphics: PLATON (Spek, 2009); software used to prepare material for publication: SHELXTL (Sheldrick, 2008).

Supplementary data and figures for this paper are available from the IUCr electronic archives (Reference: LH2821).

\section{References}

Bruker (2007). SAINT-Plus and SMART. Bruker AXS Inc., Madison, Wisconsin, USA.

Christoforou, A. M., Marzilli, P. A. \& Marzilli, L. G. (2006). Inorg. Chem. 45, 6771-6781.

Corradini, R., Dossena, A., Galaverna, G., Marchelli, R., Panagia, A. \& Sarto, G. (1997). J. Org. Chem. 62, 6283-6289.

Corradini, R., Dossena, A., Marchelli, R., Panagia, A., Sartor, G., Saviano, M., Lombardi, A. \& Pavone, V. (1996). Chem. Eur. J. 2, 373-381.

Flack, H. D. (1983). Acta Cryst. A39, 876-881.

Sheldrick, G. M. (1997). SADABS. University of Göttingen, Germany.

Sheldrick, G. M. (2008). Acta Cryst. A64, 112-122.

Spek, A. L. (2009). Acta Cryst. D65, 148-155. 


\section{supporting information}

Acta Cryst. (2009). E65, o1452 [doi:10.1107/S160053680901962X]

\section{N-(2-Aminoethyl)-5-(dimethylamino)naphthalene-1-sulfonamide}

\section{Shi-lei Zhang, Bi-lin Zhao, Zhen-hong Su, Xian-you Xia and Yong Zhang}

\section{S1. Comment}

The dansyl (5-(dimethylamino)naphthalene-1-sulfonyl) group has been widely used as a fluorophore in the design of fluorescent probes. Recently many fluorescent ligands bearing dansyl group have been reported (Corradini et al., 1996,1997; Christoforou et al., 2006). We are interested in preparing fluorescent ligands that are expected to bind to hydrophobic sites in proteins or membranes. With this mind, the title compound, (I), was prepared and we report the crystal stucture herein.

In the molecule (Fig. 1), atoms N1 and S1 are located approximately in the naphthalene ring plane with their deviations being 0.078 and $0.084 \AA$, respectively. The $\mathrm{N} 2-\mathrm{C} 14-\mathrm{C} 15-\mathrm{N} 3$ torsion angle of $-53.6(4)^{\circ}$ indicates a coiled conformation for the aminoethyl group. In the crystal structure (Fig.2), intermolecular $\mathrm{N}-\mathrm{H} \cdots \mathrm{N}$ and weak $\mathrm{C}-\mathrm{H} \cdots \mathrm{O}$ hydrogen bonds link molecules into one-dimensional chains along [001].

\section{S2. Experimental}

Compound (I) was synthesized according to a literature procedure (Corradini et al., 1996). Single crystals suitable for Xray diffraction were obtained by slow evaporation of a dichloromethane solution of (I) at room temperature.

\section{S3. Refinement}

All carbon bound $\mathrm{H}$ atoms were placed in their idealized positions [ $C-H($ methyl $)=0.96 \AA$ and $\mathrm{C}-\mathrm{H}($ aromatic $)=0.93 \AA]$ and included in the refinement in the riding-model approximation, with $U_{\text {iso }}($ methyl $\mathrm{H})=1.5 U_{\text {eq }}(\mathrm{C})$ and $U_{\text {iso }}(\operatorname{aromatic~} \mathrm{H})=$ $1.2 U_{\mathrm{eq}}(\mathrm{C})$. Hydrogen atoms bonded to nitrogen atoms were found in the difference Fourier maps and refined with the constraints of $\mathrm{N}-\mathrm{H}=0.869(\AA)$ and $U_{\text {iso }}(\mathrm{H})=1.2 U_{\text {eq }}(\mathrm{N})$. 


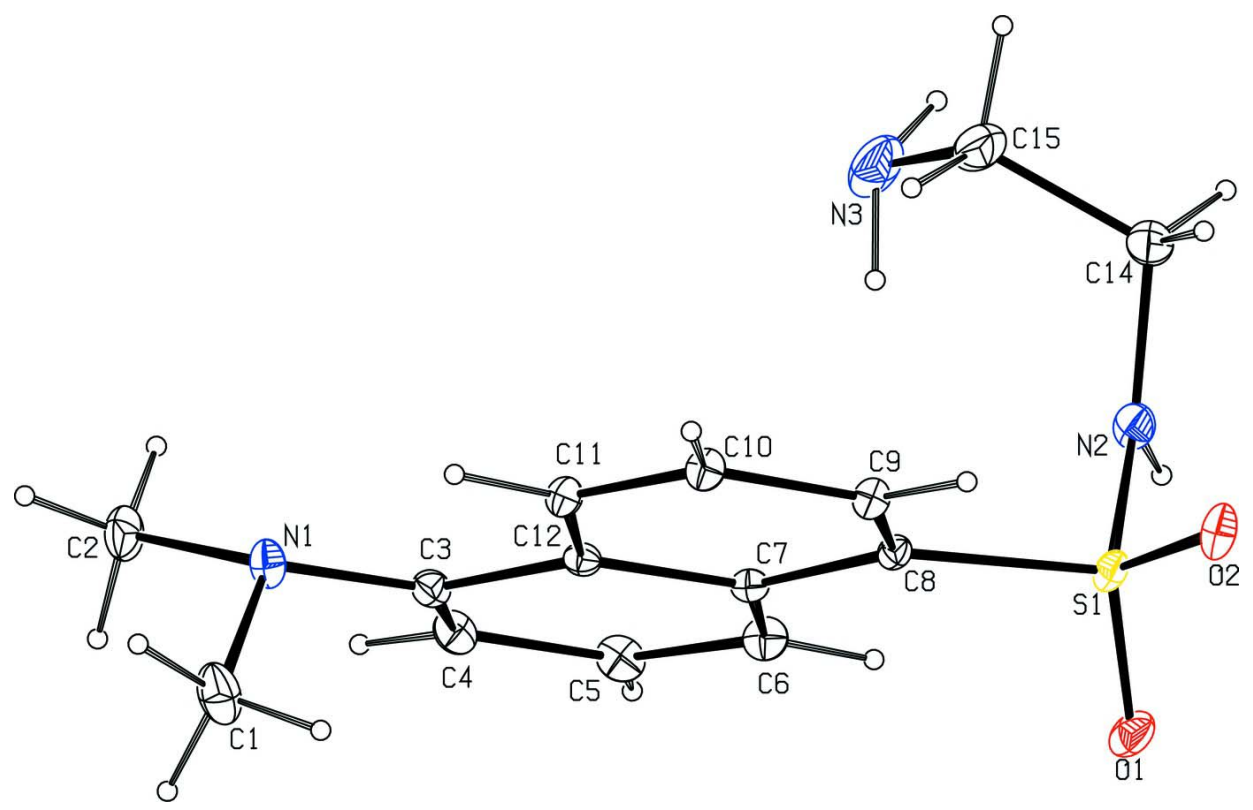

\section{Figure 1}

The molecular structure of (I), with displacement ellipsoids drawn at the 50\% probability level.

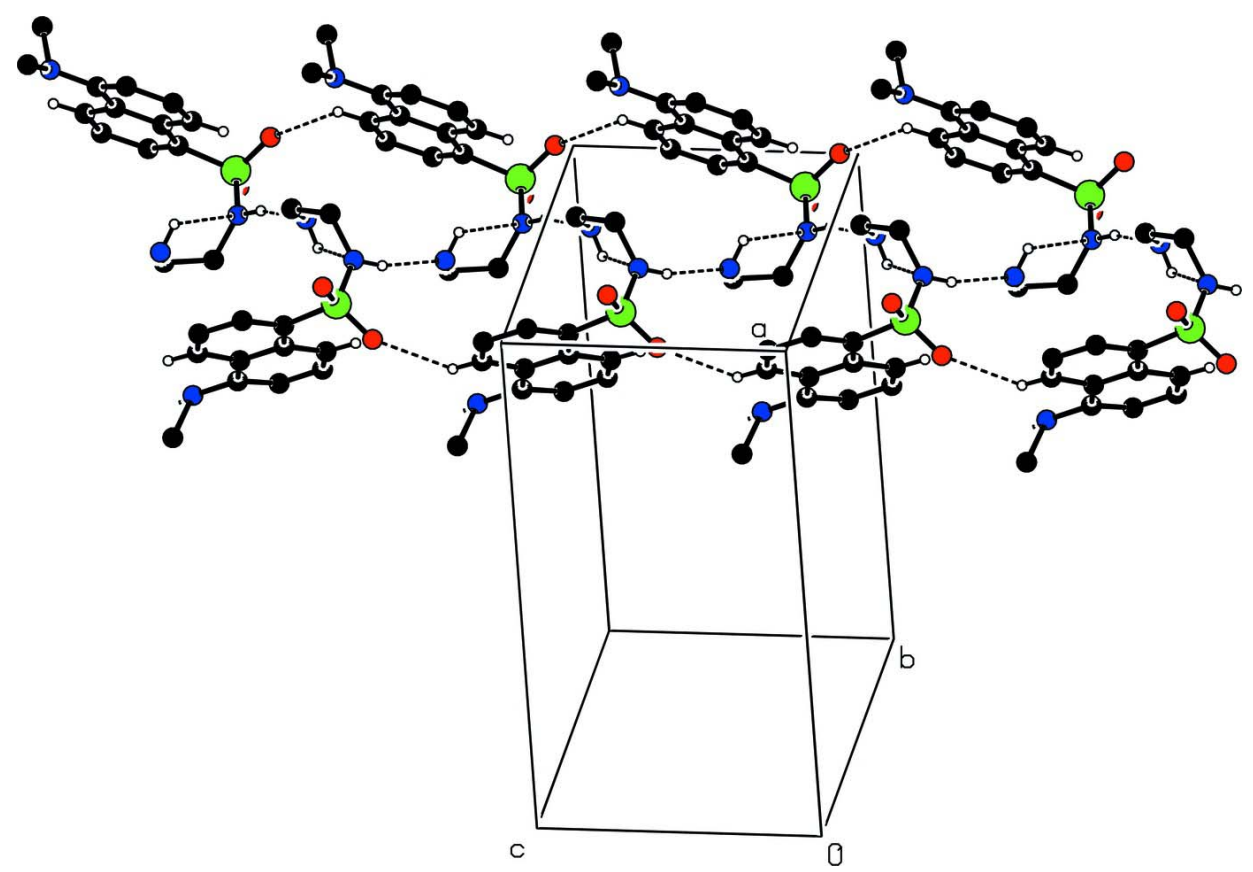

Figure 2

Part of the crystal structure of (I) showing weak hydrogen bonds as dashed lines. Only $\mathrm{H}$ atoms involved in hydrogen bonds are shown. 


\section{$N$-(2-Aminoethyl)-5-(dimethylamino)naphthalene-1-sulfonamide}

Crystal data

$\mathrm{C}_{14} \mathrm{H}_{19} \mathrm{~N}_{3} \mathrm{O}_{2} \mathrm{~S}$

$M_{r}=293.38$

Orthorhombic, Pna2

Hall symbol: P 2c $-2 \mathrm{n}$

$a=15.5221(15) \AA$

$b=11.5423(11) \AA$

$c=8.1360(8) \AA$

$V=1457.7(2) \AA^{3}$

$Z=4$

Data collection

Bruker SMART CCD

diffractometer

Radiation source: fine-focus sealed tube

Graphite monochromator

$\varphi$ and $\omega$ scans

Absorption correction: multi-scan

(SADABS; Sheldrick, 1997)

$T_{\min }=0.956, T_{\max }=0.956$

Refinement

Refinement on $F^{2}$

Least-squares matrix: full

$R\left[F^{2}>2 \sigma\left(F^{2}\right)\right]=0.041$

$w R\left(F^{2}\right)=0.107$

$S=1.11$

3140 reflections

192 parameters

1 restraint

Primary atom site location: structure-invariant direct methods

Secondary atom site location: difference Fourier map
$F(000)=624$

$D_{\mathrm{x}}=1.337 \mathrm{Mg} \mathrm{m}^{-3}$

Mo $K \alpha$ radiation, $\lambda=0.71073 \AA$

Cell parameters from 4085 reflections

$\theta=2.2-28.0^{\circ}$

$\mu=0.23 \mathrm{~mm}^{-1}$

$T=298 \mathrm{~K}$

Block, colorless

$0.20 \times 0.20 \times 0.20 \mathrm{~mm}$

7478 measured reflections

3140 independent reflections

3012 reflections with $I>2 \sigma(I)$

$R_{\text {int }}=0.029$

$\theta_{\text {max }}=27.0^{\circ}, \theta_{\min }=2.2^{\circ}$

$h=-19 \rightarrow 13$

$k=-14 \rightarrow 14$

$l=-10 \rightarrow 10$

Hydrogen site location: inferred from neighbouring sites

$\mathrm{H}$ atoms treated by a mixture of independent and constrained refinement

$w=1 /\left[\sigma^{2}\left(F_{\mathrm{o}}{ }^{2}\right)+(0.0621 P)^{2}+0.0989 P\right]$ where $P=\left(F_{\mathrm{o}}^{2}+2 F_{\mathrm{c}}^{2}\right) / 3$

$(\Delta / \sigma)_{\max }<0.001$

$\Delta \rho_{\max }=0.24 \mathrm{e} \AA^{-3}$

$\Delta \rho_{\min }=-0.25$ e $\AA^{-3}$

Absolute structure: Flack (1983), 1332 Friedel pairs

Absolute structure parameter: -0.03 (8)

Special details

Geometry. All e.s.d.'s (except the e.s.d. in the dihedral angle between two 1.s. planes) are estimated using the full covariance matrix. The cell e.s.d.'s are taken into account individually in the estimation of e.s.d.'s in distances, angles and torsion angles; correlations between e.s.d.'s in cell parameters are only used when they are defined by crystal symmetry. An approximate (isotropic) treatment of cell e.s.d.'s is used for estimating e.s.d.'s involving 1.s. planes.

Refinement. Refinement of $F^{2}$ against ALL reflections. The weighted $R$-factor $w R$ and goodness of fit $S$ are based on $F^{2}$, conventional $R$-factors $R$ are based on $F$, with $F$ set to zero for negative $F^{2}$. The threshold expression of $F^{2}>\sigma\left(F^{2}\right)$ is used only for calculating $R$-factors (gt) etc. and is not relevant to the choice of reflections for refinement. $R$-factors based on $F^{2}$ are statistically about twice as large as those based on $F$, and $R$ - factors based on ALL data will be even larger.

Fractional atomic coordinates and isotropic or equivalent isotropic displacement parameters $\left(\AA^{2}\right)$

\begin{tabular}{lllll}
\hline & $x$ & $y$ & $z$ & $U_{\text {iso }} * / U_{\text {eq }}$ \\
\hline C1 & $0.6783(2)$ & $0.2592(3)$ & $1.2600(5)$ & $0.0711(9)$ \\
H1A & 0.6195 & 0.2620 & 1.2236 & $0.107^{*}$ \\
H1B & 0.6797 & 0.2519 & 1.3775 & $0.107^{*}$
\end{tabular}




$\begin{array}{lllll}\text { H1C } & 0.7065 & 0.1937 & 1.2111 & 0.107^{*} \\ \text { C2 } & 0.67195(17) & 0.4676(3) & 1.2579(4) & 0.0615(7) \\ \text { H2A } & 0.7030 & 0.5368 & 1.2300 & 0.092^{*} \\ \text { H2B } & 0.6616 & 0.4659 & 1.3742 & 0.092^{*} \\ \text { H2C } & 0.6180 & 0.4665 & 1.2004 & 0.092^{*} \\ \text { C3 } & 0.75553(12) & 0.36601(19) & 1.0498(3) & 0.0381(5) \\ \text { C4 } & 0.72410(14) & 0.43583(19) & 0.9255(3) & 0.0451(5) \\ \text { H4 } & 0.6796 & 0.4874 & 0.9477 & 0.054^{*} \\ \text { C5 } & 0.75833(15) & 0.4299(2) & 0.7670(3) & 0.0480(6) \\ \text { H5 } & 0.7350 & 0.4767 & 0.6853 & 0.058^{*} \\ \text { C6 } & 0.82454(15) & 0.3580(2) & 0.7281(3) & 0.0445(5) \\ \text { H6 } & 0.8451 & 0.3547 & 0.6208 & 0.053^{*} \\ \text { C7 } & 0.86251(12) & 0.28748(17) & 0.8530(3) & 0.0323(4) \\ \text { C8 } & 0.93538(13) & 0.21356(16) & 0.8264(3) & 0.0309(4) \\ \text { C9 } & 0.96977(14) & 0.14947(18) & 0.9519(3) & 0.0366(4) \\ \text { H9 } & 1.0158 & 0.0999 & 0.9311 & 0.044^{*} \\ \text { C10 } & 0.93643(14) & 0.15783(18) & 1.1106(3) & 0.0379(4) \\ \text { H10 } & 0.9610 & 0.1151 & 1.1954 & 0.045^{*} \\ \text { C11 } & 0.86816(12) & 0.22823(16) & 1.1418(3) & 0.0363(4) \\ \text { H11 } & 0.8473 & 0.2345 & 1.2486 & 0.044^{*} \\ \text { C12 } & 0.82814(12) & 0.29230(16) & 1.0143(3) & 0.0322(4) \\ \text { C14 } & 1.10343(19) & 0.3731(3) & 0.6827(4) & 0.0689(9) \\ \text { H14A } & 1.1354 & 0.4225 & 0.6080 & 0.083^{*} \\ \text { H14B } & 1.1400 & 0.3079 & 0.7107 & 0.083^{*} \\ \text { C15 } & 1.0839(2) & 0.4406(3) & 0.8375(5) & 0.0833(11) \\ \text { H15A } & 1.0609 & 0.3874 & 0.9188 & 0.100^{*} \\ \text { H15B } & 1.1375 & 0.4712 & 0.8805 & 0.100^{*} \\ \text { N1 } & 0.72278(13) & 0.36630(17) & 1.2108(3) & 0.0467(5) \\ \text { O1 } & 0.92413(13) & 0.18510(17) & 0.5084(2) & 0.0558(5) \\ \text { O2 } & 1.05529(12) & 0.12231(15) & 0.6510(2) & 0.0553(4) \\ \text { N2 } & 1.02722(14) & 0.33007(18) & 0.5987(3) & 0.0495(5) \\ \text { H2D } & 1.0054(19) & 0.358(3) & 0.508(5) & 0.059^{*} \\ \text { N3 } & 1.0237(3) & 0.5358(3) & 0.8182(5) & 0.0993(13) \\ \text { H3A } & 0.980(3) & 0.499(5) & 0.773(8) & 0.119^{*} \\ \text { H3B } & 1.044(3) & 0.588(4) & 0.752(7) & 0.119^{*} \\ \text { S1 } & 0.98740(3) & 0.20448(4) & 0.63277(8) & 0.03840(15) \\ & & & & \end{array}$

Atomic displacement parameters $\left(\AA^{2}\right)$

\begin{tabular}{lllllll}
\hline & $U^{11}$ & $U^{22}$ & $U^{33}$ & $U^{12}$ & $U^{13}$ & $U^{23}$ \\
\hline C1 & $0.0693(17)$ & $0.0655(18)$ & $0.079(2)$ & $0.0010(15)$ & $0.0311(15)$ & $0.0156(17)$ \\
C2 & $0.0547(14)$ & $0.0663(17)$ & $0.0635(18)$ & $0.0210(13)$ & $0.0108(12)$ & $-0.0083(14)$ \\
C3 & $0.0340(10)$ & $0.0365(11)$ & $0.0439(12)$ & $0.0001(8)$ & $-0.0005(9)$ & $-0.0006(9)$ \\
C4 & $0.0377(10)$ & $0.0406(12)$ & $0.0570(14)$ & $0.0071(9)$ & $-0.0008(10)$ & $0.0082(11)$ \\
C5 & $0.0472(12)$ & $0.0486(13)$ & $0.0482(14)$ & $0.0062(10)$ & $-0.0097(10)$ & $0.0171(11)$ \\
C6 & $0.0533(12)$ & $0.0461(12)$ & $0.0343(11)$ & $-0.0003(10)$ & $-0.0053(9)$ & $0.0120(10)$ \\
C7 & $0.0331(9)$ & $0.0315(9)$ & $0.0323(10)$ & $-0.0037(7)$ & $-0.0041(8)$ & $0.0023(8)$ \\
C8 & $0.0369(10)$ & $0.0294(9)$ & $0.0264(10)$ & $-0.0018(8)$ & $0.0014(8)$ & $-0.0008(8)$
\end{tabular}


supporting information

\begin{tabular}{lllllll} 
C9 & $0.0418(10)$ & $0.0344(11)$ & $0.0336(10)$ & $0.0061(8)$ & $-0.0015(9)$ & $0.0015(8)$ \\
C10 & $0.0450(10)$ & $0.0387(10)$ & $0.0299(11)$ & $0.0066(8)$ & $-0.0045(8)$ & $0.0077(8)$ \\
C11 & $0.0434(10)$ & $0.0367(9)$ & $0.0286(9)$ & $0.0018(7)$ & $0.0003(9)$ & $0.0037(9)$ \\
C12 & $0.0328(9)$ & $0.0303(10)$ & $0.0335(10)$ & $-0.0049(7)$ & $-0.0015(8)$ & $0.0030(8)$ \\
C14 & $0.0647(16)$ & $0.0609(16)$ & $0.081(3)$ & $-0.0208(14)$ & $0.0173(15)$ & $-0.0189(15)$ \\
C15 & $0.099(2)$ & $0.077(2)$ & $0.075(2)$ & $-0.034(2)$ & $0.012(2)$ & $-0.0264(19)$ \\
N1 & $0.0462(10)$ & $0.0458(11)$ & $0.0482(11)$ & $0.0089(9)$ & $0.0133(9)$ & $0.0015(9)$ \\
O1 & $0.0778(12)$ & $0.0595(11)$ & $0.0302(8)$ & $-0.0089(9)$ & $-0.0003(8)$ & $-0.0045(8)$ \\
O2 & $0.0729(10)$ & $0.0498(9)$ & $0.0432(9)$ & $0.0162(8)$ & $0.0174(9)$ & $-0.0028(8)$ \\
N2 & $0.0641(13)$ & $0.0425(10)$ & $0.0420(14)$ & $-0.0092(9)$ & $0.0122(9)$ & $0.0037(9)$ \\
N3 & $0.158(4)$ & $0.0527(18)$ & $0.087(2)$ & $-0.0261(19)$ & $0.036(2)$ & $-0.0248(16)$ \\
S1 & $0.0538(3)$ & $0.0341(2)$ & $0.0274(2)$ & $-0.00129(19)$ & $0.0066(3)$ & $-0.0034(3)$ \\
\hline
\end{tabular}

Geometric parameters $\left(\AA,{ }^{\circ}\right)$

\begin{tabular}{|c|c|c|c|}
\hline $\mathrm{C} 1-\mathrm{N} 1$ & $1.472(3)$ & $\mathrm{C} 8-\mathrm{S} 1$ & $1.773(2)$ \\
\hline $\mathrm{C} 1-\mathrm{H} 1 \mathrm{~A}$ & 0.9600 & $\mathrm{C} 9-\mathrm{C} 10$ & $1.395(3)$ \\
\hline $\mathrm{C} 1-\mathrm{H} 1 \mathrm{~B}$ & 0.9600 & $\mathrm{C} 9-\mathrm{H} 9$ & 0.9300 \\
\hline $\mathrm{C} 1-\mathrm{H} 1 \mathrm{C}$ & 0.9600 & $\mathrm{C} 10-\mathrm{C} 11$ & $1.359(3)$ \\
\hline $\mathrm{C} 2-\mathrm{N} 1$ & $1.461(3)$ & $\mathrm{C} 10-\mathrm{H} 10$ & 0.9300 \\
\hline $\mathrm{C} 2-\mathrm{H} 2 \mathrm{~A}$ & 0.9600 & $\mathrm{C} 11-\mathrm{C} 12$ & $1.417(3)$ \\
\hline $\mathrm{C} 2-\mathrm{H} 2 \mathrm{~B}$ & 0.9600 & $\mathrm{C} 11-\mathrm{H} 11$ & 0.9300 \\
\hline $\mathrm{C} 2-\mathrm{H} 2 \mathrm{C}$ & 0.9600 & $\mathrm{C} 14-\mathrm{N} 2$ & $1.454(4)$ \\
\hline $\mathrm{C} 3-\mathrm{C} 4$ & $1.382(3)$ & $\mathrm{C} 14-\mathrm{C} 15$ & $1.511(5)$ \\
\hline $\mathrm{C} 3-\mathrm{N} 1$ & $1.405(3)$ & $\mathrm{C} 14-\mathrm{H} 14 \mathrm{~A}$ & 0.9700 \\
\hline $\mathrm{C} 3-\mathrm{C} 12$ & $1.441(3)$ & C14-H14B & 0.9700 \\
\hline $\mathrm{C} 4-\mathrm{C} 5$ & $1.396(3)$ & $\mathrm{C} 15-\mathrm{N} 3$ & $1.450(5)$ \\
\hline $\mathrm{C} 4-\mathrm{H} 4$ & 0.9300 & C15-H15A & 0.9700 \\
\hline $\mathrm{C} 5-\mathrm{C} 6$ & $1.358(3)$ & C15-H15B & 0.9700 \\
\hline $\mathrm{C} 5-\mathrm{H} 5$ & 0.9300 & $\mathrm{O} 1-\mathrm{S} 1$ & $1.4278(19)$ \\
\hline $\mathrm{C} 6-\mathrm{C} 7$ & $1.429(3)$ & $\mathrm{O} 2-\mathrm{S} 1$ & $1.4255(18)$ \\
\hline $\mathrm{C} 6-\mathrm{H} 6$ & 0.9300 & $\mathrm{~N} 2-\mathrm{S} 1$ & $1.600(2)$ \\
\hline $\mathrm{C} 7-\mathrm{C} 12$ & $1.417(3)$ & $\mathrm{N} 2-\mathrm{H} 2 \mathrm{D}$ & $0.87(3)$ \\
\hline $\mathrm{C} 7-\mathrm{C} 8$ & $1.433(3)$ & $\mathrm{N} 3-\mathrm{H} 3 \mathrm{~A}$ & $0.88(5)$ \\
\hline $\mathrm{C} 8-\mathrm{C} 9$ & $1.369(3)$ & $\mathrm{N} 3-\mathrm{H} 3 \mathrm{~B}$ & $0.87(5)$ \\
\hline $\mathrm{N} 1-\mathrm{C} 1-\mathrm{H} 1 \mathrm{~A}$ & 109.5 & $\mathrm{C} 9-\mathrm{C} 10-\mathrm{H} 10$ & 119.9 \\
\hline $\mathrm{N} 1-\mathrm{C} 1-\mathrm{H} 1 \mathrm{~B}$ & 109.5 & $\mathrm{C} 10-\mathrm{C} 11-\mathrm{C} 12$ & $121.1(2)$ \\
\hline $\mathrm{H} 1 \mathrm{~A}-\mathrm{C} 1-\mathrm{H} 1 \mathrm{~B}$ & 109.5 & $\mathrm{C} 10-\mathrm{C} 11-\mathrm{H} 11$ & 119.4 \\
\hline $\mathrm{N} 1-\mathrm{C} 1-\mathrm{H} 1 \mathrm{C}$ & 109.5 & $\mathrm{C} 12-\mathrm{C} 11-\mathrm{H} 11$ & 119.4 \\
\hline $\mathrm{H} 1 \mathrm{~A}-\mathrm{C} 1-\mathrm{H} 1 \mathrm{C}$ & 109.5 & $\mathrm{C} 11-\mathrm{C} 12-\mathrm{C} 7$ & $119.47(18)$ \\
\hline $\mathrm{H} 1 \mathrm{~B}-\mathrm{C} 1-\mathrm{H} 1 \mathrm{C}$ & 109.5 & $\mathrm{C} 11-\mathrm{C} 12-\mathrm{C} 3$ & $120.3(2)$ \\
\hline $\mathrm{N} 1-\mathrm{C} 2-\mathrm{H} 2 \mathrm{~A}$ & 109.5 & $\mathrm{C} 7-\mathrm{C} 12-\mathrm{C} 3$ & $120.20(18)$ \\
\hline $\mathrm{N} 1-\mathrm{C} 2-\mathrm{H} 2 \mathrm{~B}$ & 109.5 & $\mathrm{~N} 2-\mathrm{C} 14-\mathrm{C} 15$ & $113.9(3)$ \\
\hline $\mathrm{H} 2 \mathrm{~A}-\mathrm{C} 2-\mathrm{H} 2 \mathrm{~B}$ & 109.5 & $\mathrm{~N} 2-\mathrm{C} 14-\mathrm{H} 14 \mathrm{~A}$ & 108.8 \\
\hline $\mathrm{N} 1-\mathrm{C} 2-\mathrm{H} 2 \mathrm{C}$ & 109.5 & $\mathrm{C} 15-\mathrm{C} 14-\mathrm{H} 14 \mathrm{~A}$ & 108.8 \\
\hline $\mathrm{H} 2 \mathrm{~A}-\mathrm{C} 2-\mathrm{H} 2 \mathrm{C}$ & 109.5 & $\mathrm{~N} 2-\mathrm{C} 14-\mathrm{H} 14 \mathrm{~B}$ & 108.8 \\
\hline $\mathrm{H} 2 \mathrm{~B}-\mathrm{C} 2-\mathrm{H} 2 \mathrm{C}$ & 109.5 & $\mathrm{C} 15-\mathrm{C} 14-\mathrm{H} 14 \mathrm{~B}$ & 108.8 \\
\hline
\end{tabular}




\begin{tabular}{|c|c|c|c|}
\hline $\mathrm{C} 4-\mathrm{C} 3-\mathrm{N} 1$ & $123.6(2)$ & $\mathrm{H} 14 \mathrm{~A}-\mathrm{C} 14-\mathrm{H} 14 \mathrm{~B}$ & 107.7 \\
\hline $\mathrm{C} 4-\mathrm{C} 3-\mathrm{C} 12$ & $118.3(2)$ & $\mathrm{N} 3-\mathrm{C} 15-\mathrm{C} 14$ & $115.5(3)$ \\
\hline $\mathrm{N} 1-\mathrm{C} 3-\mathrm{C} 12$ & $118.11(19)$ & $\mathrm{N} 3-\mathrm{C} 15-\mathrm{H} 15 \mathrm{~A}$ & 108.4 \\
\hline $\mathrm{C} 3-\mathrm{C} 4-\mathrm{C} 5$ & $120.8(2)$ & $\mathrm{C} 14-\mathrm{C} 15-\mathrm{H} 15 \mathrm{~A}$ & 108.4 \\
\hline $\mathrm{C} 3-\mathrm{C} 4-\mathrm{H} 4$ & 119.6 & $\mathrm{~N} 3-\mathrm{C} 15-\mathrm{H} 15 \mathrm{~B}$ & 108.4 \\
\hline $\mathrm{C} 5-\mathrm{C} 4-\mathrm{H} 4$ & 119.6 & $\mathrm{C} 14-\mathrm{C} 15-\mathrm{H} 15 \mathrm{~B}$ & 108.4 \\
\hline $\mathrm{C} 6-\mathrm{C} 5-\mathrm{C} 4$ & $122.3(2)$ & $\mathrm{H} 15 \mathrm{~A}-\mathrm{C} 15-\mathrm{H} 15 \mathrm{~B}$ & 107.5 \\
\hline $\mathrm{C} 6-\mathrm{C} 5-\mathrm{H} 5$ & 118.9 & $\mathrm{C} 3-\mathrm{N} 1-\mathrm{C} 2$ & $116.2(2)$ \\
\hline $\mathrm{C} 4-\mathrm{C} 5-\mathrm{H} 5$ & 118.9 & $\mathrm{C} 3-\mathrm{N} 1-\mathrm{C} 1$ & $114.9(2)$ \\
\hline $\mathrm{C} 5-\mathrm{C} 6-\mathrm{C} 7$ & $119.6(2)$ & $\mathrm{C} 2-\mathrm{N} 1-\mathrm{C} 1$ & $110.3(2)$ \\
\hline $\mathrm{C} 5-\mathrm{C} 6-\mathrm{H} 6$ & 120.2 & $\mathrm{C} 14-\mathrm{N} 2-\mathrm{S} 1$ & $122.8(2)$ \\
\hline $\mathrm{C} 7-\mathrm{C} 6-\mathrm{H} 6$ & 120.2 & $\mathrm{C} 14-\mathrm{N} 2-\mathrm{H} 2 \mathrm{D}$ & $126(2)$ \\
\hline $\mathrm{C} 12-\mathrm{C} 7-\mathrm{C} 6$ & $118.73(19)$ & $\mathrm{S} 1-\mathrm{N} 2-\mathrm{H} 2 \mathrm{D}$ & $109(2)$ \\
\hline $\mathrm{C} 12-\mathrm{C} 7-\mathrm{C} 8$ & $117.42(17)$ & $\mathrm{C} 15-\mathrm{N} 3-\mathrm{H} 3 \mathrm{~A}$ & $100(3)$ \\
\hline $\mathrm{C} 6-\mathrm{C} 7-\mathrm{C} 8$ & $123.8(2)$ & $\mathrm{C} 15-\mathrm{N} 3-\mathrm{H} 3 \mathrm{~B}$ & $111(3)$ \\
\hline $\mathrm{C} 9-\mathrm{C} 8-\mathrm{C} 7$ & $121.08(19)$ & $\mathrm{H} 3 \mathrm{~A}-\mathrm{N} 3-\mathrm{H} 3 \mathrm{~B}$ & $111(5)$ \\
\hline $\mathrm{C} 9-\mathrm{C} 8-\mathrm{S} 1$ & $116.94(16)$ & $\mathrm{O} 2-\mathrm{S} 1-\mathrm{O} 1$ & $118.56(12)$ \\
\hline $\mathrm{C} 7-\mathrm{C} 8-\mathrm{S} 1$ & $121.94(15)$ & $\mathrm{O} 2-\mathrm{S} 1-\mathrm{N} 2$ & $109.58(12)$ \\
\hline $\mathrm{C} 8-\mathrm{C} 9-\mathrm{C} 10$ & $120.57(19)$ & $\mathrm{O} 1-\mathrm{S} 1-\mathrm{N} 2$ & $106.55(12)$ \\
\hline $\mathrm{C} 8-\mathrm{C} 9-\mathrm{H} 9$ & 119.7 & $\mathrm{O} 2-\mathrm{S} 1-\mathrm{C} 8$ & $106.46(10)$ \\
\hline $\mathrm{C} 10-\mathrm{C} 9-\mathrm{H} 9$ & 119.7 & $\mathrm{O} 1-\mathrm{S} 1-\mathrm{C} 8$ & $109.01(10)$ \\
\hline $\mathrm{C} 11-\mathrm{C} 10-\mathrm{C} 9$ & $120.2(2)$ & $\mathrm{N} 2-\mathrm{S} 1-\mathrm{C} 8$ & $106.04(10)$ \\
\hline $\mathrm{C} 11-\mathrm{C} 10-\mathrm{H} 10$ & 119.9 & & \\
\hline $\mathrm{N} 1-\mathrm{C} 3-\mathrm{C} 4-\mathrm{C} 5$ & $-178.7(2)$ & $\mathrm{C} 4-\mathrm{C} 3-\mathrm{C} 12-\mathrm{C} 11$ & $173.49(19)$ \\
\hline $\mathrm{C} 12-\mathrm{C} 3-\mathrm{C} 4-\mathrm{C} 5$ & $3.9(3)$ & $\mathrm{N} 1-\mathrm{C} 3-\mathrm{C} 12-\mathrm{C} 11$ & $-4.0(3)$ \\
\hline $\mathrm{C} 3-\mathrm{C} 4-\mathrm{C} 5-\mathrm{C} 6$ & $-1.4(4)$ & $\mathrm{C} 4-\mathrm{C} 3-\mathrm{C} 12-\mathrm{C} 7$ & $-3.6(3)$ \\
\hline $\mathrm{C} 4-\mathrm{C} 5-\mathrm{C} 6-\mathrm{C} 7$ & $-1.5(4)$ & $\mathrm{N} 1-\mathrm{C} 3-\mathrm{C} 12-\mathrm{C} 7$ & $178.89(18)$ \\
\hline $\mathrm{C} 5-\mathrm{C} 6-\mathrm{C} 7-\mathrm{C} 12$ & $1.7(3)$ & $\mathrm{N} 2-\mathrm{C} 14-\mathrm{C} 15-\mathrm{N} 3$ & $-53.6(4)$ \\
\hline $\mathrm{C} 5-\mathrm{C} 6-\mathrm{C} 7-\mathrm{C} 8$ & $-176.60(19)$ & $\mathrm{C} 4-\mathrm{C} 3-\mathrm{N} 1-\mathrm{C} 2$ & $-18.4(3)$ \\
\hline $\mathrm{C} 12-\mathrm{C} 7-\mathrm{C} 8-\mathrm{C} 9$ & $0.6(3)$ & $\mathrm{C} 12-\mathrm{C} 3-\mathrm{N} 1-\mathrm{C} 2$ & $159.0(2)$ \\
\hline $\mathrm{C} 6-\mathrm{C} 7-\mathrm{C} 8-\mathrm{C} 9$ & $178.9(2)$ & $\mathrm{C} 4-\mathrm{C} 3-\mathrm{N} 1-\mathrm{C} 1$ & $112.6(3)$ \\
\hline $\mathrm{C} 12-\mathrm{C} 7-\mathrm{C} 8-\mathrm{S} 1$ & $-177.20(14)$ & $\mathrm{C} 12-\mathrm{C} 3-\mathrm{N} 1-\mathrm{C} 1$ & $-70.0(3)$ \\
\hline $\mathrm{C} 6-\mathrm{C} 7-\mathrm{C} 8-\mathrm{S} 1$ & $1.1(3)$ & $\mathrm{C} 15-\mathrm{C} 14-\mathrm{N} 2-\mathrm{S} 1$ & $-91.9(3)$ \\
\hline $\mathrm{C} 7-\mathrm{C} 8-\mathrm{C} 9-\mathrm{C} 10$ & $-2.3(3)$ & $\mathrm{C} 14-\mathrm{N} 2-\mathrm{S} 1-\mathrm{O} 2$ & $-40.1(2)$ \\
\hline $\mathrm{S} 1-\mathrm{C} 8-\mathrm{C} 9-\mathrm{C} 10$ & $175.56(16)$ & $\mathrm{C} 14-\mathrm{N} 2-\mathrm{S} 1-\mathrm{O} 1$ & $-169.58(19)$ \\
\hline $\mathrm{C} 8-\mathrm{C} 9-\mathrm{C} 10-\mathrm{C} 11$ & $1.3(3)$ & $\mathrm{C} 14-\mathrm{N} 2-\mathrm{S} 1-\mathrm{C} 8$ & $74.4(2)$ \\
\hline $\mathrm{C} 9-\mathrm{C} 10-\mathrm{C} 11-\mathrm{C} 12$ & $1.5(3)$ & $\mathrm{C} 9-\mathrm{C} 8-\mathrm{S} 1-\mathrm{O} 2$ & $3.06(19)$ \\
\hline $\mathrm{C} 10-\mathrm{C} 11-\mathrm{C} 12-\mathrm{C} 7$ & $-3.2(3)$ & $\mathrm{C} 7-\mathrm{C} 8-\mathrm{S} 1-\mathrm{O} 2$ & $-179.09(16)$ \\
\hline $\mathrm{C} 10-\mathrm{C} 11-\mathrm{C} 12-\mathrm{C} 3$ & $179.68(19)$ & $\mathrm{C} 9-\mathrm{C} 8-\mathrm{S} 1-\mathrm{O} 1$ & $132.05(17)$ \\
\hline $\mathrm{C} 6-\mathrm{C} 7-\mathrm{C} 12-\mathrm{C} 11$ & $-176.28(19)$ & $\mathrm{C} 7-\mathrm{C} 8-\mathrm{S} 1-\mathrm{O} 1$ & $-50.11(19)$ \\
\hline $\mathrm{C} 8-\mathrm{C} 7-\mathrm{C} 12-\mathrm{C} 11$ & $2.1(3)$ & $\mathrm{C} 9-\mathrm{C} 8-\mathrm{S} 1-\mathrm{N} 2$ & $-113.59(18)$ \\
\hline $\mathrm{C} 6-\mathrm{C} 7-\mathrm{C} 12-\mathrm{C} 3$ & $0.8(3)$ & $\mathrm{C} 7-\mathrm{C} 8-\mathrm{S} 1-\mathrm{N} 2$ & $64.25(19)$ \\
\hline $\mathrm{C} 8-\mathrm{C} 7-\mathrm{C} 12-\mathrm{C} 3$ & $179.24(17)$ & & \\
\hline
\end{tabular}




\section{supporting information}

Hydrogen-bond geometry $\left(\AA,{ }^{\circ}\right)$

\begin{tabular}{lllll}
\hline$D-\mathrm{H} \cdots A$ & $D-\mathrm{H}$ & $\mathrm{H} \cdots A$ & $D \cdots A$ & $D-\mathrm{H} \cdots A$ \\
\hline $\mathrm{C} 6-\mathrm{H} 6 \cdots \mathrm{O} 1$ & 0.93 & 2.48 & $3.093(3)$ & 123 \\
$\mathrm{~N} 3-\mathrm{H} 3 A \cdots \mathrm{N} 2$ & $0.88(5)$ & $2.52(6)$ & $2.972(4)$ & $113(4)$ \\
$\mathrm{C} 11-\mathrm{H} 11 \cdots \mathrm{O} 1^{\mathrm{i}}$ & 0.93 & 2.49 & $3.146(3)$ & 128 \\
$\mathrm{~N} 2-\mathrm{H} 2 D \cdots \mathrm{N} 3^{3 i}$ & $0.87(3)$ & $2.02(4)$ & $2.869(4)$ & $163(3)$ \\
\hline
\end{tabular}

Symmetry codes: (i) $x, y, z+1$; (ii) $-x+2,-y+1, z-1 / 2$. 